\title{
Thiophene-based oligomers with high photo and electroluminescence efficiencies across the entire visible range
}

\author{
Giovanna Barbarella*a ${ }^{\mathrm{a}}$, Laura Favaretto ${ }^{\mathrm{a}}$, Giovanna Sotgiu ${ }^{\mathrm{a}}$, Massimo Zambianchi ${ }^{\mathrm{a}}$, \\ Giuseppe Gigli ${ }^{\mathrm{b}}$, Roberto Cingolani ${ }^{\mathrm{b}}$ \\ ${ }^{a}$ Consiglio Nazionale Ricerche, I.Co.C.E.A, Via Gobetti 101, 40129 Bologna, Italy \\ ${ }^{\mathrm{b}}$ Istituto Nazionale di Fisica della Materia, Dipartimento Ingegneria Innovazione \\ Università di Lecce, Via Arnesano, 73100 Lecce, Italy
}

\begin{abstract}
We present a new class of thiophene-based molecular materials with high solid-state photoluminescence efficiencies across the entire visible range. We show that full color tunability may be achieved, including white and purple, by binary blends of these materials, according to CIE standards. With some of these materials efficient electroluminescent devices were fabricated and characterized.
\end{abstract}

Keywords: thiophene oligomers, electron affinity, photoluminescence, CIE diagram, electroluminescence, UV-Vis

\section{INTRODUCTION}

Organic molecules with high photoluminescence efficiencies are increasingly in demand for application in many research fields from fabricating electroluminescent devices for flat-panel display technology to probing biologically important compounds. ${ }^{1,2}$

Thiophene-based oligomers, which have well defined structures and monodispersed chain lengths, are currently receiving increasing attention owing to their chemical stability and easy functionalization which allow for the construction of molecular architectures of great diversity and properties. ${ }^{3}$ These compounds are known for their good electrical properties. However, the type of molecular organization in the solid state that leads to efficient charge transport is detrimental to photoluminescence efficiency. Conventional thiophene oligomers have low photoluminescence efficiencies in the solid state since they tend to assume coplanar conformations and self-assemble in H-type aggregates. The analysis of single crystal X-ray structures has shown that this kind of supramolecular organization is the result of a large number of weak and directional hydrogen bonding interactions between the $\mathrm{C}-\mathrm{H}$ groups and the sulfur atoms or the $\mathrm{C}-\mathrm{H}$ groups and the $\pi$-electron system of the thienyl rings. ${ }^{4}$

* E-mail: barbarella@area.bo.cnr.it 
We present a new class of thiophene-based oligomers in which the functionalization of the thienyl sulfur with oxygen atoms introduces other kinds of intermolecular interactions in the solid state which counteract the tendency to organize in parallel layers ${ }^{4}$ and then decreases the number of possible non radiative patterns for energy relaxation from the singlet excited state. Moreover, this type of functionalization also brings about a large increase in electron affinity, as deduced from electrochemical reduction potentials measured by cyclic voltammetry.

\section{RESULTS}

Figure 1 illustrates the effect of functionalization in engineering the solid-state photoluminescence efficiency of a series of thiophene pentamers. It is seen that the solid-state photoluminescence efficiency varies from $2 \%$ for conventional quinquethiophene to $48 \%$ for the pentamer containing the rigid dithienothiophene core, a value which is more than one order of magnitude larger than the quantum efficiency generally measured in unmodified conventional oligothiophenes.

Single crystal X-ray diffraction studies on a structurally homogeneous series of oligo(thiophene-S,S-dioxides) allowed to demonstrate that the high-solid state photoluminescence efficiency of these compounds is strictly related to a different type of molecular self-assembly in the solid state. ${ }^{5}$ The presence of the thienyl-S,S-dioxide moieties in the backbone leads to intermolecular interactions which counteract the tendency of conventional thiophene oligomers to assume coplanar conformations and self-assemble in H-type aggregates. The high solid-state photoluminescence efficiency of oligo(thiophene-S,S-dioxides) is the result of increased separation between chain backbones and the existence of a finite angle between the long molecular axes.

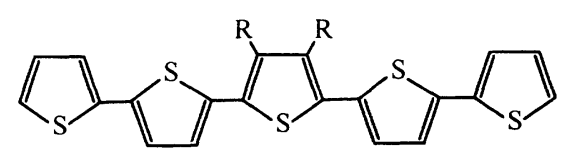

$2 \%$

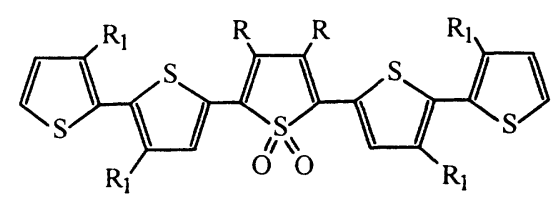

$37 \%$

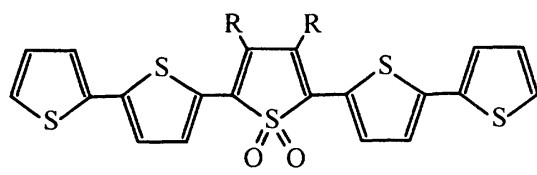

$12 \%$

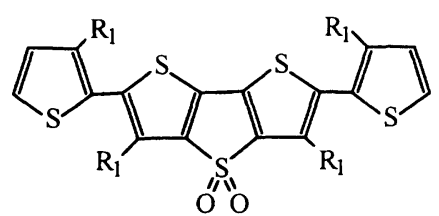

$48 \%$

Figure 1. Molecular structure $\left[\mathrm{R}=\left(\mathrm{CH}_{2}\right)_{5} \mathrm{CH}_{3}, \mathrm{R}_{1}=\mathrm{CH}_{3}\right]$ and absolute solid-state photoluminescence efficiencies $(\eta \%)$ of selected thiophene pentamers 
We have synthesized a large variety of oligothiophene-S,S-dioxides and shown that the solid-state photoluminescence

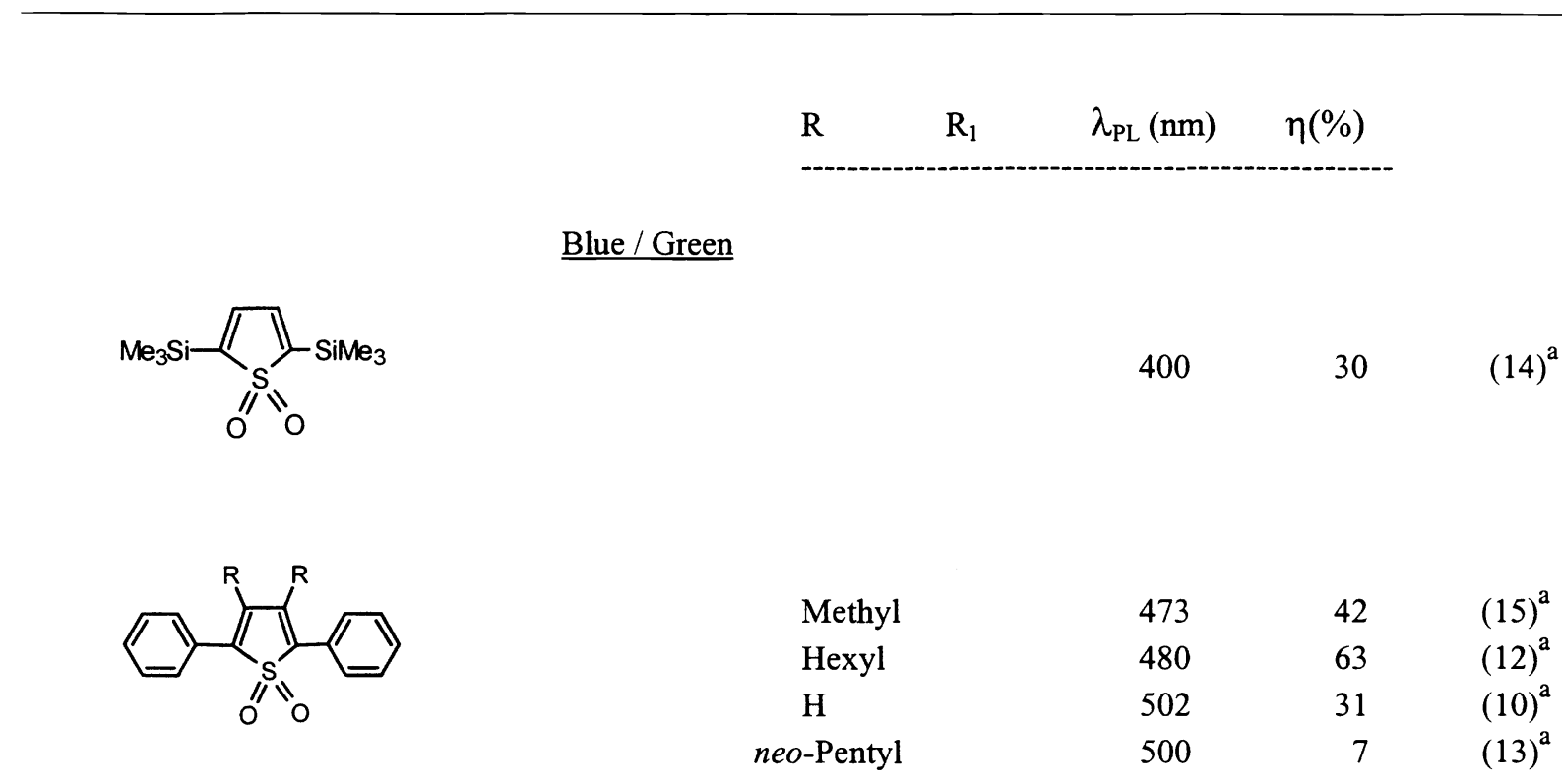

\section{Green / Yellow}<smiles>[R]C1=C(c2cccs2)S(=O)(=O)C(c2cccs2)=C1[R]</smiles>

$\begin{array}{llll}\text { Hexyl } & 526 & 45 & (9)^{\mathrm{a}} \\ \text { Methyl } & 556 & 33 & (8)^{\mathrm{a}} \\ \text { Phenyl } & 573 & 30 & (7)^{\mathrm{a}}\end{array}$<smiles>[R]C1=C(c2ccc(-c3ccccc3)cc2)S(=O)(=O)C(c2ccc(-c3ccccc3)cc2)=C1[R]</smiles>

Hexyl

525

70

$(11)^{\mathrm{a}}$

\section{Orange / Red}<smiles>[R]C1=C(c2cc([R])c(-c3sccc3[R8])s2)S(=O)(=O)C(c2sc(-c3sccc3[R])cc2[R])=C1[R]</smiles>

$\begin{array}{lllll}\text { Hexyl } & \text { Methyl } & 590 & 37 & (6)^{\text {a }} \\ \text { Hexyl } & \text { Cyclohexyl } & 620 & 23 & \\ \text { Methyl } & \text { Methyl } & 632 & 13 & \\ \text { Hexyl } & \text { H } & 660 & 13 & (2)^{\text {a }}\end{array}$

Figure 2. Solid-state photoluminescence frequencies $\left(\lambda_{\mathrm{PL}}, \mathrm{nm}\right)$ and absolute solid-state photoluminescence efficiencies $(\eta \%)$ of selected oligo(thiophene-S,S-dioxides).

a. Numbering corresponding to figures 3-6 
frequency under UV excitation of these compounds can be finely tuned across the entire visible range with a great variety of shades and with good solid-state photoluminescence efficiencies in all color ranges. ${ }^{6}$ We were able to obtain solid-state photoluminescence efficiences up to 70\%, as shown in Figure 2.

The PL efficiencies reported in figures 1 and 2 were measured using an integrating sphere and are relative to microcrystalline powders. Very similar values were also obtained with spin-coated films of the same compounds.

These high photoluminescence efficiency oligo(thiopheneS,S-dioxide) cover almost the entire spectrum of colors according to the standards of the Commission International de l'Eclairage (CIE), as shown in Figure 3.

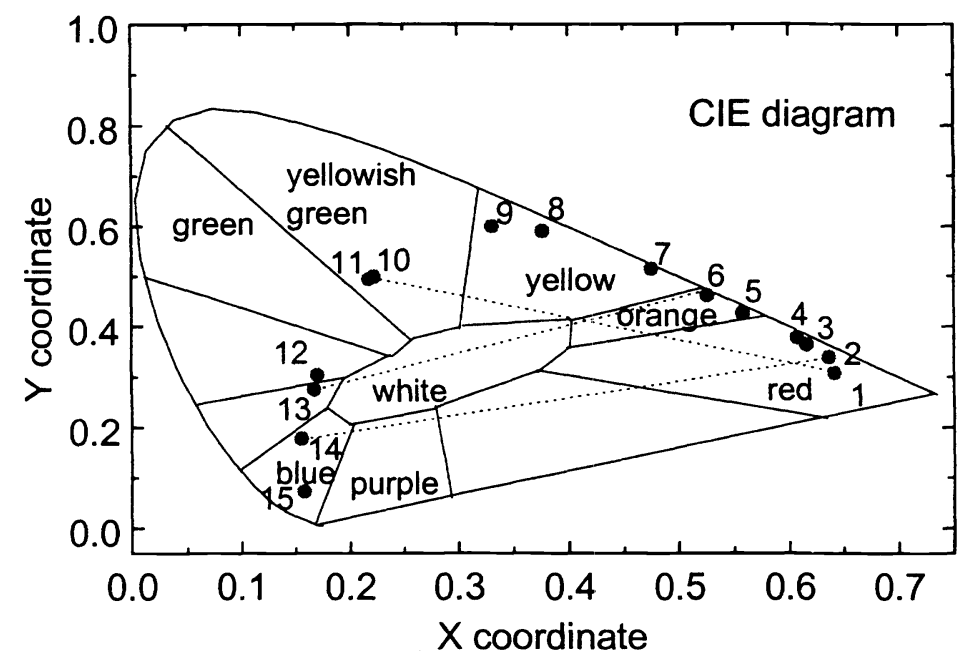

Figure 3. Spectral distribution of oligo(thiophene-S,S-dioxides) according to the CIE standards.

Moreover, we have prepared binary blends by spin coating thiophene solutions in chloroform of these modified, high photoluminescence efficiency, oligomers. Binary blends resulting in purple, red and white colors were prepared (see 
figures 4 and 5). The white color of the blend could be tuned by varying the relative amount of the constituent compounds, from a reddish white to a greenish white along the dashed line connecting compounds of Figure 3 . We were able to show that the emission spectrum of the blend is determined by the Forster transfer when the energy separation between the HOMO-LUMO gap of the constituting molecules is smaller than $0.56 \mathrm{eV}$, while for larger energy separation, the blend emission is just given by the superposition of the emission spectra of the constituting molecules. ${ }^{6}$

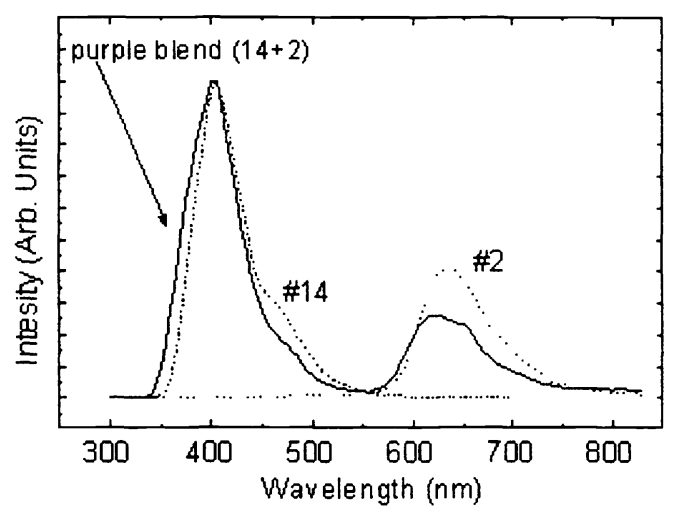

Fig. 4. Emission spectrum of the purple blend at room temperature (continuous line). The dashed line and the dotted line represent the emission spectra of the constituting molecules, normalized to their relative quantum efficiency.

Fig. 5. Emission spectrum of the white blend (continuous line) and of the constituent molecules (dashed and dotted curves, normalized to the relative quantum efficiency). 
As mentioned above, the functionalization of the thienyl sulfur with oxygen leads not only to increased PL efficiencies in the solid state but also to increased electron affinities. ${ }^{7}$ This is illustrated in figure 6 , showing that the frontier orbital energies of selected oligomers containing the thienyl-S,S-dioxide unit are markedly displaced towards more negative values (with respect to the vacuum level) compared to those of corresponding unsubstituted phenylene (a) or thienylene (b) oligomers, indicating the increase of both the electron affinities and the ionization potentials in these kinds of materials. The figure also reports the work functions of aluminum and calcium and the work function of ITO which are generally used as the cathode and the anode materials, respectively, in the fabrication of electroluminescent devices.

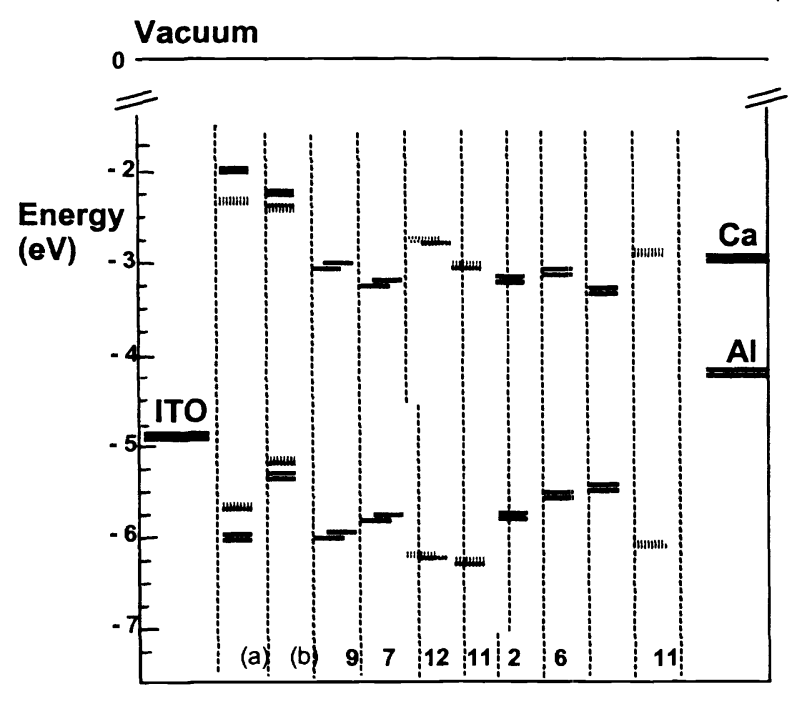

Figure 6. Ionization potentials and electron affinities of selected oligomers containing the thienyl-S,S-dioxide moiety obtained from electrochemical oxidation and reduction peak potentials. (a) ter- and hexaphenylene (b) ter- and sexithiophene

Conventional thienylene and phenylene oligomers are $p$-type semiconductor materials with low electron affinities. Finding a kind of functionalization capable of increasing their electron affinity is a necessary step to make these materials more suitable for the fabrication of electroluminescent devices. Indeed, the greater the electron affinity of the light emitting material is, the smaller is the energy barrier between the LUMO and the Fermi level of the metal used as the cathode, leading to better electron injection and increased device performance. 
Electroluminescent diodes with good efficiencies - at least one order of magnitude greater than the best reported so far in the literature for conventional thiophene oligomers - have been fabricated. EL efficiences up to $1.2 \mathrm{Cd} / \mathrm{A}$, luminance up to $200 \mathrm{Cd} / \mathrm{m} 2$ and turn voltage down to $1.9 \mathrm{~V}$ were obtained. ${ }^{8-10}$

Good electroluminescence tunability was demonstrated using the compounds of figures 1 and 2, as shown in Figure 6. Stable and low turn on voltage devices were fabricated by spin coating the active layer, with EL efficiencies up to $0.1 \%$. The LEDs were prepared by spin coating onto indium-tin-oxide (ITO) coated glass substrates first a hole transporting material and then a chloroform solution of the pure active material. The cathodes $(\mathrm{Ca} / \mathrm{Al})$ were deposited by thermal evaporation. Device characterization was carried out in air atmosphere. ${ }^{11}$

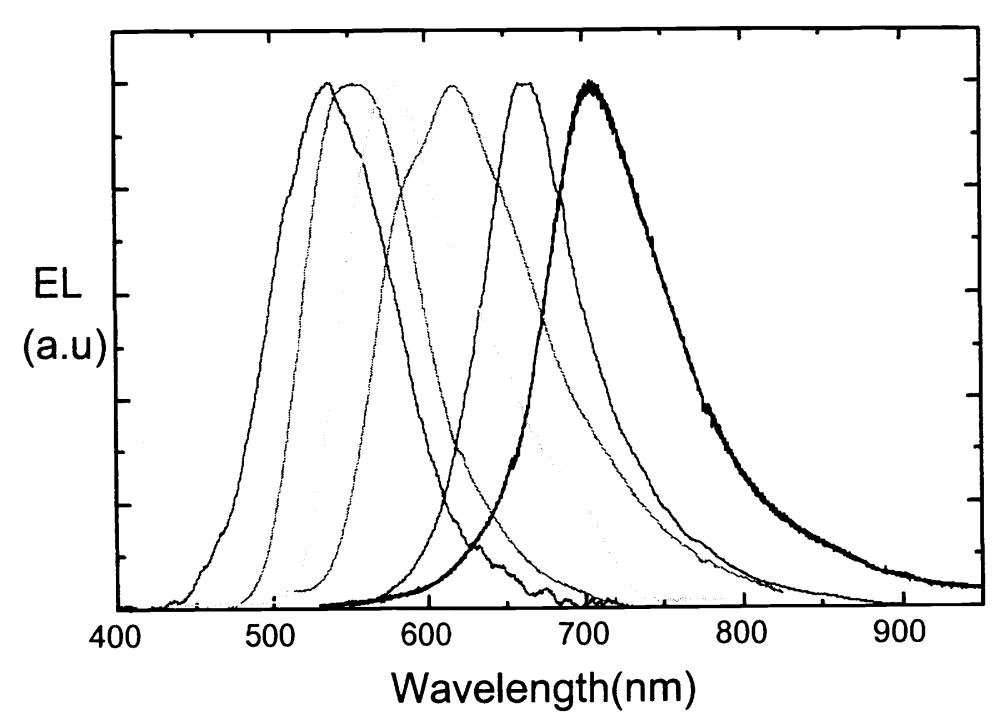

Figure 7. Electroluminescence spectra of selected oligothiophene-S,S-dioxides containing one central thienyl-S,S-dioxide unity. The lowest wavelength spectrum is that of compound 11 (Table 2) while the highest wavelength one pertains to the heptamer having one central dihexyl-thienyl-S,S-dioxide unity.

\section{CONCLUSION}

We have shown that a series of conjugated oligomers exhibiting unprecedented fluorescence efficiency in the solid state and covering the entire visible range can be obtained using the thienyl-S,S-dioxide moiety as a building block. 
As a consequence of the increased electron affinity also brought about by the presence of the oxygen atoms, the energy levels of conjugated oligomers containing the thienyl-S,S-dioxide moiety provide a closer match to the work function of cathode contact metals. Increased solid-state photoluminescence efficiencies and electron affinities make these compounds suitable for LED technology. Highly efficient LED devices were fabricated.

\section{REFERENCES}

1. J.R. Sheats, H. Antoniadis, M. Hueschen, W. Leonard, J. Miller, R. Moon, D. Roitman, A. Stocking, Science 1996, 273, 884.

2. Bruchez, Jr., M. Moronne, P. Gin, S. Weiss, A.P. Alivisatos. Science 1998, $281,2013$.

3. K. Müllen, G. Wegner, Eds. Electronic Materials: The Oligomer Approach.. Wiley-VCH: New York, 1998.

4. G. Barbarella, M. Zambianchi, L. Antolini, P. Ostoja, P. Maccagnani, A. Bongini, E. A. Marseglia, E. Tedesco, G. Gigli, R. Cingolani. J. Am. Chem. Soc. 1999, 121, 8920.

5. L. Antolini, E. Tedesco, G. Barbarella, L. Favaretto, G. Sotgiu, M. Zambianchi, D. Casarini, G. Gigli, R. Cingolani. J. Am. Chem. Soc. 2000, 000.

6. M. Anni, G. Gigli, V. Paladini, R. Cingolani, G. Barbarella, L. Favaretto, G. Sotgiu, M. Zambianchi. Appl. Phys. Lett., 2000, 000.

7. G. Barbarella, L. Favaretto, M. Zambianchi, O. Pudova, C. Arbizzani, A. Bongini, M. Mastragostino. Adv Mater. 1998, 10, 551.

8. G. Gigli, G. Barbarella, L. Favaretto, F. Cacialli, R. Cingolani. Appl. Phys. Lett. 1999, 75, 439.

9. G. Barbarella, L. Favaretto, G. Sotgiu, M. Zambianchi, V. Fattori, M. Cocchi, F. Cacialli, G. Gigli, R. Cingolani. Adv.Mater. 1999, 11, 1375.

10. V. Fattori, M. Cocchi, P. Di Marco, G. Giro, G. Barbarella, G. Sotgiu. Synthetic Metals 2000, 111, 83.

11. G.Gigli, M.Anni, M.Theander, R.Cingolani, G.Barbarella, L. Favaretto, O.Inganäs. International Conference on Science and Technology of Synthetic Metals, 15 - 21 July 2000, Gastein, Austria. 